This item was submitted to Loughborough's Research Repository by the author.

Items in Figshare are protected by copyright, with all rights reserved, unless otherwise indicated.

\title{
Illumination modelling of a mobile device environment for effective use in driving mobile apps
}

PLEASE CITE THE PUBLISHED VERSION

http://dx.doi.org/10.1117/12.2177087

PUBLISHER

(c) SPIE

VERSION

AM (Accepted Manuscript)

LICENCE

CC BY-NC-ND 4.0

\section{REPOSITORY RECORD}

Al-Marhoubi, Asmaa H.A., Sara Saravi, Eran A. Edirisinghe, and Helmut E. Bez. 2019. "Illumination Modelling of a Mobile Device Environment for Effective Use in Driving Mobile Apps". figshare.

https://hdl.handle.net/2134/21953. 


\title{
Illumination Modelling of a Mobile Device Environment for Effective use in Driving Mobile Apps
}

\author{
Asmaa H. Marhoubi, Sara Saravi, Eran A. Edirisinghe, Helmut H. Bez \\ Department of Computer Science, Loughborough University, United Kingdom
}

\begin{abstract}
The present generation of Ambient Light Sensors (ALS) of a mobile handheld device suffer from two practical shortcomings. The ALSs are narrow angle, i.e. they respond effectively only within a narrow angle of operation and there is a latency of operation. As a result mobile applications that operate based on the ALS readings could perform sub-optimally especially when operated in environments with non-uniform illumination. The applications will either adopt with unacceptable levels of latency or/and may demonstrate a discrete nature of operation. In this paper we propose a framework to predict the ambient illumination of an environment in which a mobile device is present. The predictions are based on an illumination model that is developed based on a small number of readings taken during an application calibration stage. We use a machine learning based approach in developing the models. Five different regression models were developed, implemented and compared based on Polynomial, Gaussian, Sum of Sine, Fourier and Smoothing Spline functions. Approaches to remove noisy data, missing values and outliers were used prior to the modelling stage to remove their negative effects on modelling. The prediction accuracy for all models were found to be above 0.99 when measured using R-Squared test with the best performance being from Smoothing Spline. In this paper we will discuss mathematical complexity of each model and investigate how to make compromises in finding the best model.
\end{abstract}

Keywords: Ambient Light Sensor, Mobile Handheld Devices, Illumination Modelling, Regression Analysis, Machine Learning.

\section{INTRODUCTION}

\subsection{Introduction}

The Ambient Light Sensor (ALS) [1] of a mobile device is a powerful device integrated within all handheld mobile devices including mobile phones, tablets, laptops and event some of the desktop computer and television displays. They produce a reading that is proportional to the lux reading of the ambient illumination [2] of a device from which the intensity of illumination that falls on the display screen is deduced. In some devices this sensor reading is also used to estimate the display's backlight level.

Both the ambient illumination and backlight [3] values are used to estimate the illumination in an environment in which the mobile device operates allowing applications that present especially image and video content to the users to best respond to the changes of environment. The applications can thus be designed to automatically change the screen's brightness allowing the content to be better viewable under extreme changes of screen illumination and reflections. These automatic adjustments including the control of the screen backlight intensity also provides an additional advantage in saving battery life.

Most mobile device manufacturers and third party application providers to the industry have produced different approaches for the above purpose. Some of these approaches are known publically but others are protected technology knowhow of the relevant manufacturers and technology providers. A number of attempts have also been taken in literature by the research community to address the above with the key focus varying from proving the best quality of experience to the users to the most effective approach to saving battery life. In [4], Bai et.al. discussed how to reduce the power consumption of an LCD display. They proposed an approach to control the LCD brightness in a way that makes it comfortable to the eye considering the lighting in an environment. A FLC (Fuzzy Logic Controller) was used to control the backlight brightness of the LCD panel aided by the ALS of a notebook computer. The availability of the light sensor to sense the environment was utilised to automatically adjust the backlight brightness in order to reduce the power consumption of the LCD

Image Sensing Technologies: Materials, Devices, Systems, and Applications II, edited by Nibir K. Dhar, Achyut K. Dutta, Proc. of SPIE Vol. 9481, 94810R

(C) 2015 SPIE · CCC code: $0277-786 X / 15 / \$ 18 \cdot$ doi: 10.1117/12.2177087 
panel. In [5], Ma et. al. proposed a method to improve the automatic brightness control on Android handheld devices in low illumination. The front camera and a face detection algorithm were used to calculate the contrast ratio between a user's face and background to determine the optimum screen brightness. However in this approach the use of the front camera has an adverse effect on the battery consumption of the device. In [6], Mailloux et. al. addressed the issue of automatically adjusting the screen and keypad light on mobile devices. The median of five consecutive ALS reading stored in a buffer were used to control the screen and keypad brightness levels demonstrating the ability of the method to effectively manage battery power consumption. In [7], Dalen et. al. proposed the use of light sensor capable of measuring both the intensity level and the colour temperature of the environment to drive an electronic controller that predicted whether a mobile device was being used more likely within an artificially lit indoor environment or a more naturally lit outdoor environment. In [8], Robinson et. al. proposed a method to automatically adjust the brightness of a display screen of a mobile device by making use of both the light sensor and the orientation sensor. The orientation sensor uses the device's accelerometer to detect the orientation of the device. The proposed approach used the sensor readings from the light sensor and the orientation sensor to enable the effective adjustment the backlight intensity resulting directly in the reduction of power consumption. In [1], Cho et. al. studied in detail the battery consumption of a mobile device that is due to the required backlighting of a display. His research revealed that the backlight accounts to an average $30 \%$ of battery consumption in a mobile phones normal daily usage.

The literature review conducted within the research remit of this paper, summarized above, shows that the key research focus has been to propose ideas to effectively manage the backlight intensity of a device based on the ALS readings on a mobile device. Unfortunately this work is largely limited by two practical shortcomings of the present generation of ALS devices, namely, the narrow angle and latency of operation. Most sensors that are in use today operates effectively only within a narrow angle of operation (8-10 degrees from the normal) and has a noticeable latency of response that can undermine the performance of any existing technology that controls the backlight level based on the ALS readings, aimed at saving battery power. However if the environmental illumination can be modelled, the a priori knowledge can be effectively used in overcoming both the narrow angle of operation and also latency of ALS response. For example, from the laws of optical physics it is known that the illumination intensity from a point light source varies proportionally to the inverse square of the distance from the light source [9]. This will result in the intensity variation of the ALS sensor of a mobile device moved directly under a fixed point light source to behave in a predictable manner, i.e. illustrating the shape of a normal/Gaussian distribution. When the illumination of the environment becomes more complex, i.e the presence of two or more lighting sources, diffused light sources, different orientations of lighting sources etc., the illumination modelling will require more complex mathematical analysis. However these complex environments can be ultimately modelled using more simple models capable of extending the modelling of illumination from s ingle light sources. In light of the above research gap in this paper we provide the results of a preliminary study that first characterizes the typical performance/response of an ALS sensor in the presence of a single point light source and two point light sources and then uses mathematical regression to accurately predict an illumination model following a machine learning approach. This research will provide a vital insight into the practicality of ALS sensor behavior correction and its possible use in making present and future mobile application more adaptive to the environments in which they are being used.

For clarity of presentation this paper is divided into several sections. Apart from this section which provided an insight into the problem domain and existing literature that enabled the identification of the research gaps, section 2 provides the reader the experimental methodology followed to capture data. Section 3 provides the results and a detailed analysis. Finally section 5 concludes with an insight into future research and potential applications of the presented research.

\section{EXPERIMENTAL METHODOLOGY}

The modelling of illumination in the area surrounding a mobile device requires the investigation of the possibility of using various mathematical functions as successful models. As the illumination intensity of a point is inversely proportional square of the it's distance from a point light source our initial predictions were that functions involving sine and cosine terms, including simple sine functions, their powers, sum-of-sine functions, 
Fourier and Gaussian distributions will be good fits. Further we decided to investigate the use of a polynomial function and also a smoothing spline [10].

The modelling of the illumination of an environment requires that the above models are trained on some known intensity values of known points in the 3D space. As mentioned before the key focus of our current research attempt is to evaluate the possibility of modelling more fundamental illumination models from which more complex models can be deduced later, for more complex environments. To this extent the experiments were conducted to model the illumination that results from one point light source and two separate point light sources.

The experiments were conducted indoors in a dark room where all usual light sources were turned off. A wooden surfaced, non-reflective table of $97.8 \mathrm{~cm}$ in length, $67.3 \mathrm{~cm}$ in width and $65 \mathrm{~cm}$ in height was kept at the centre of the room. A LED point light source was used for the experiment; it was hung on a tripod at approximately $75 \mathrm{~cm}$ height, from the table. An Asus Nexus 7 tablet PC (version 2013) was used as the mobile that includes an ALS. To enable capturing the ALS sensor values, an Android software application was developed and installed.

The following points summaries the steps that were used for capturing the sensor values:

- All light sources were turned off and further steps were taken to ensure that the room was 'dark'.

- The light source was turned on and the Nexus 7 was placed at one end of the table.

- The top part of the Nexus 7 where the ALS is located was placed at the far edge of the table and the part where pulling cable was connected was placed at the inner side of the table.

- Once the data capture software was turned on, the Nexus 7 was pulled at an average speed of 0.00014 $\mathrm{m} / \mathrm{s}$ towards the opposite end of the table and then the software was turned off.

- The Nexus 7 was connected to the computer to load the captured values of the ALS sensor readings.

A graph was drawn with the actual illumination values placed on the y-axis and time placed on the $\mathrm{x}$-axis. The plot shows a steady increment towards a peak that was achieved when the device was just beyond the point directly under light source and showed a steady decrease is illumination beyond this peak. Figure 4.1 illustrates this graph. A closer investigation of the graph reveals that the distortions are due to the slight variations of the hand-pulled speed, the latency of response of the ALS reader and possible secondary reflections. An ideal graph should indicate a bell shape given the laws of physics governing light emission from a single point light source. However figure 1 illustrates a more practically obtainable graph given the fact that mobile devices are used by humans and the sensors have limitations in latency of operation and angle of response. Hence this is the type of graph (not the ideal 'bell shape') that should be accurately modelled. 


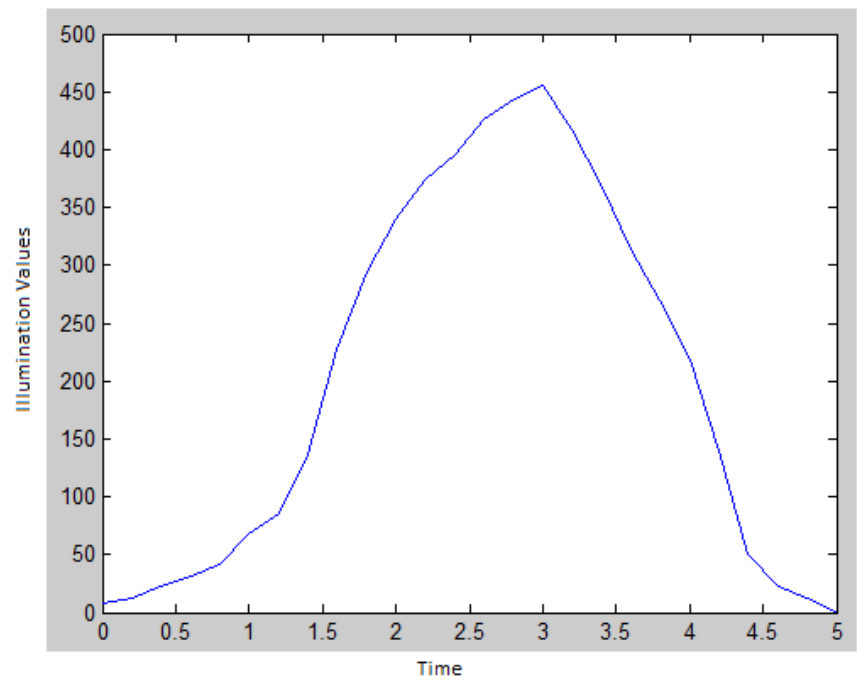

Figure 1 . The actual illumination values captured by the ALS

\section{RESULTS \& ANALYSIS}

Experiments were conducted to analyze the prediction capability of the six basic mathematical models that included, a Powers of Sine, Sums of Sine, Polynomials of different degrees, Fourier harmonics, Gaussian derivative and a Smoothing Spline. Experiments were conducted to model the illumination distributions that are caused by a single and two separate, point light sources.

\subsection{Modelling illumination due to a single light source}

Table 1 tabulates the best results obtainable by each of the basic functions and indicates the specific function that resulted in the optimum result. The comparisons are made using metrics RMSE and R-square [11].

Table 1. Comparison of the best results obtainable via each of the six different models

\begin{tabular}{|l|l|l|}
\hline Goodness of fit/Model & R-square & RMSE \\
\hline $\mathrm{f}(\mathrm{x})=\mathrm{a} \cdot \sin ^{3}(\mathrm{bx}-\mathrm{t})$ & 0.9893 & 17.96 \\
\hline $9^{\text {th }}$ Degree Polynomial & 0.9945 & 14.99 \\
\hline $8^{\text {th }}$ Fourier Harmonic & 0.9994 & 6.262 \\
\hline $4^{\text {th }}$ Gaussian Derivative & 0.999 & 6.795 \\
\hline Smoothing Spline & 1 & 0.7212 \\
\hline $4^{\text {th }}$ Sum of Sine & 0.9959 & 13.55 \\
\hline
\end{tabular}

According to the results tabulated in figure 1, the most accurate result is the Smoothing Spline. The accuracies of the $8^{\text {th }}$ Fourier harmonic and $4^{\text {th }}$ Gaussian derivative are very close to each other. The worst accuracy is shown by $f(x)=a \cdot \sin (b x-t)^{3}$. However the R-Square value still remains at 0.98 , which still indicates a significantly good fitness. 
Table 2. The Mathematical Equations of the models presented in table 1

\begin{tabular}{|c|c|}
\hline Model & Mathematical Equation \\
\hline $9^{\text {th }}$ Degree Polynomial & $\begin{array}{l}f(x)=p 1^{*} x^{\wedge} 9+p 2^{*} x^{\wedge} 8+p 3^{*} x^{\wedge} 7+p 4^{*} x^{\wedge} 6+p 5^{*} x^{\wedge} 5+p 6^{*} x^{\wedge} 4+p 7^{*} x^{\wedge} 3+ \\
p 8^{*} x^{\wedge} 2+p 9^{*} x+p 10\end{array}$ \\
\hline $8^{\text {th }}$ Fourier Harmonic & 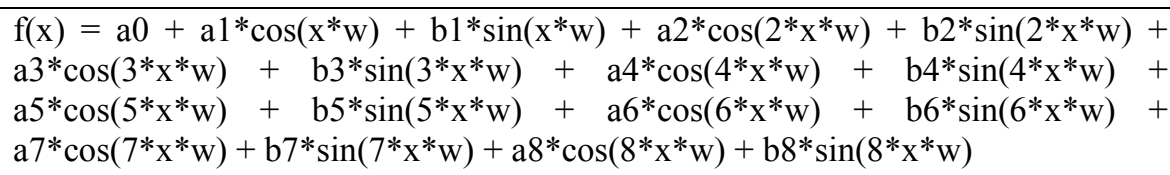 \\
\hline $4^{\text {th }}$ Gaussian Derivative & $\begin{aligned} \mathrm{f}(\mathrm{x})= & \mathrm{a} 1 * \exp \left(-((\mathrm{x}-\mathrm{b} 1) / \mathrm{c} 1)^{\wedge} 2\right)+\mathrm{a} 2 * \exp \left(-((\mathrm{x}-\mathrm{b} 2) / \mathrm{c} 2)^{\wedge} 2\right)+ \\
& \mathrm{a} 3 * \exp \left(-((\mathrm{x}-\mathrm{b} 3) / \mathrm{c} 3)^{\wedge} 2\right)+\mathrm{a} 4 * \exp \left(-((\mathrm{x}-\mathrm{b} 4) / \mathrm{c} 4)^{\wedge} 2\right)\end{aligned}$ \\
\hline Smoothing Spline & Multiple equations for each segment of the curve [12] \\
\hline $4^{\text {th }}$ Sum of Sine & $\begin{array}{l}\mathrm{f}(\mathrm{x})=\mathrm{a} 1 * \sin (\mathrm{b} 1 * \mathrm{x}+\mathrm{c} 1)+\mathrm{a}^{2} * \sin (\mathrm{b} 2 * \mathrm{x}+\mathrm{c} 2)+ \\
4 * \sin (\mathrm{b} 4 * \mathrm{x}+\mathrm{c} 4)\end{array}$ \\
\hline Sine Function & a.sin $(b x-t)^{3}$ \\
\hline
\end{tabular}

The mathematical equations of all of the best performing models are summarised in table 2. It suggests that the practicality of adopting each of these mathematical models on handheld devices should be looked at from a perspective beyond accuracy indicated by the R-Square values, namely the mathematical complexity of the equation itself. It is questionable if the hardware/computational constraints within a handheld mobile device make some of these models such as the Smoothing Spline, $8^{\text {th }}$ Fourier Harmonic etc practically viable. When more computations are required, the cost of the hardware will increase due to the supporting processing power required. Results suggest that, in particular the $4^{\text {th }}$ Sum-of-Sines and the $3^{\text {rd }}$ power of Sine functions are reasonably simple for implementation, with marginally lower accuracy figures as compared to the rest..

Table 3 tabulates the simplest mathematical models that could be implemented for each of the fundamental mathematical models considered, excluding the Smoothing Spline which was omitted due to its well-known complexity of implementation. The accuracy values still remain reasonable except for the case of a simple Sine function for which the accuracy figure is only marginally over 0.85 measured in terms of R-square value.

Table 3. Comparing the Simplest Models obtainable from each basic model tested.

\begin{tabular}{|l|l|l|}
\hline Mathematical Model & R-Square & Equation \\
\hline $4^{\text {th }}$ Polynomial degree & 0.9554 & $\mathrm{f}(\mathrm{x})=\mathrm{p} 1 * \mathrm{x}^{\wedge} 4+\mathrm{p} 2 * \mathrm{x}^{\wedge} 3+\mathrm{p} 3 * \mathrm{x}^{\wedge} 2+\mathrm{p} 4 * \mathrm{x}+\mathrm{p} 5$ \\
\hline $1^{\text {st }}$ Gaussian Derivative & 0.9882 & $\mathrm{f}(\mathrm{x})=\mathrm{a} 1 * \exp \left(-((\mathrm{x}-\mathrm{b} 1) / \mathrm{c} 1)^{\wedge} 2\right)$ \\
\hline $1^{\text {st }}$ Fourier Harmonic & 0.9789 & $\mathrm{f}(\mathrm{x})=\mathrm{a} 0+\mathrm{a} 1 * \cos \left(\mathrm{x}^{*} \mathrm{w}\right)+\mathrm{b} 1 * \sin \left(\mathrm{x}^{*} \mathrm{w}\right)$ \\
\hline $2^{\text {nd }}$ Sum of Sine & 0.9933 & $\mathrm{f}(\mathrm{x})=\mathrm{a} 1 * \sin (\mathrm{b} 1 * \mathrm{x}+\mathrm{c} 1)+\mathrm{a} 2 * \sin (\mathrm{b} 2 * \mathrm{x}+\mathrm{c} 2)$ \\
\hline Sine Function & 0.8552 & $\mathrm{f}(\mathrm{x})=\mathrm{a} * \sin (\mathrm{b} * \mathrm{x})+\mathrm{c}$ \\
\hline
\end{tabular}

Based on results tabulated in table 3 , the $2^{\text {nd }}$ Sum of Sine function seems to be the best choice overall choice. It results in the best accuracy of prediction (0.9933) for the most reasonable level of complexity of implementing two different basic Sine functions.

Figures 2 (a)-(f) illustrate the fitness of six mathematical functions tested in modeling the input illumination variation. A close visual comparison of the graphs shows that the Smoothing Spline shows the best prediction 
capability/fitness being able to fit to the input data, considering noise present in the data. The $4^{\text {th }}$ Gaussian derivative and the $8^{\text {th }}$ Fourier harmonic are also capable of tracking the imperfections significantly well. The same cannot be said about the remaining three mathematical models.
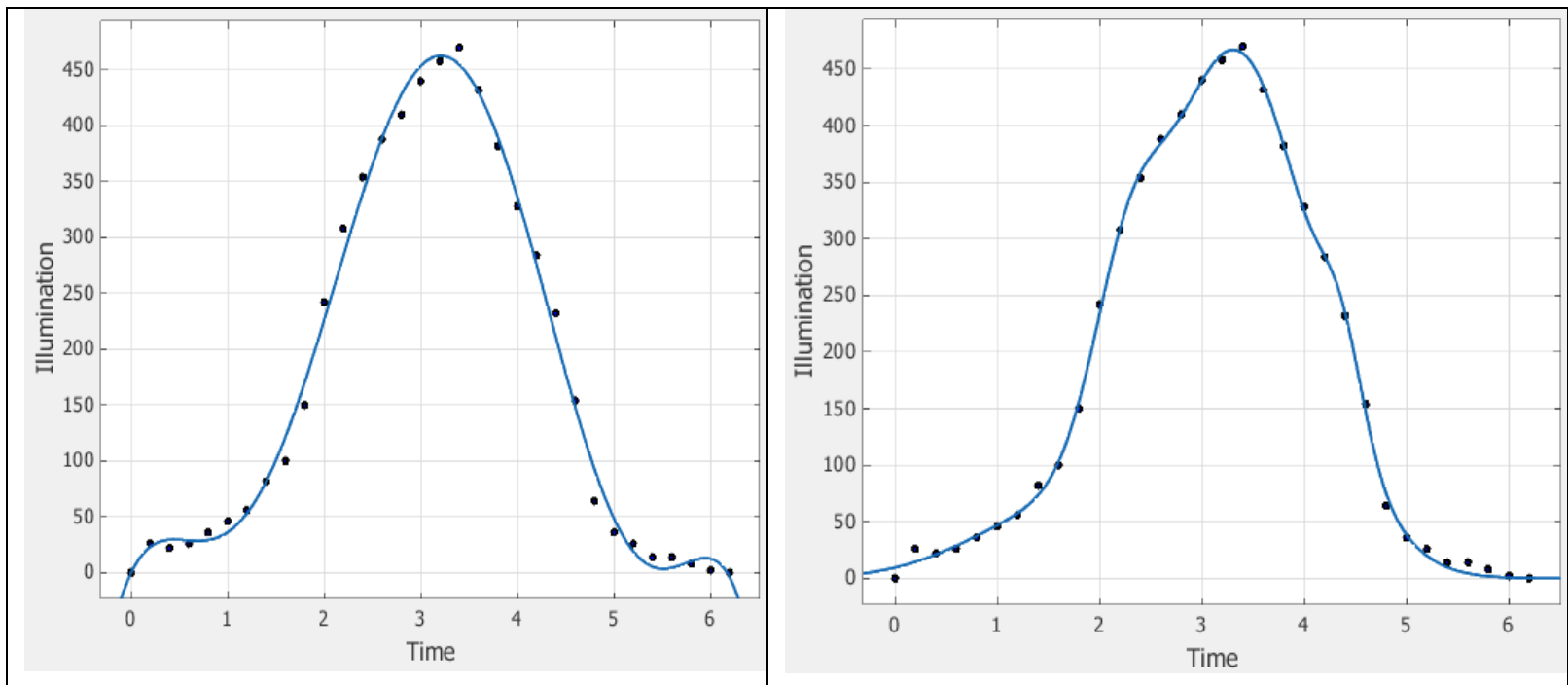

(a) $9^{\text {th }}$ Degree Polynomial

(b) $4^{\text {th }}$ Gaussian Derivative

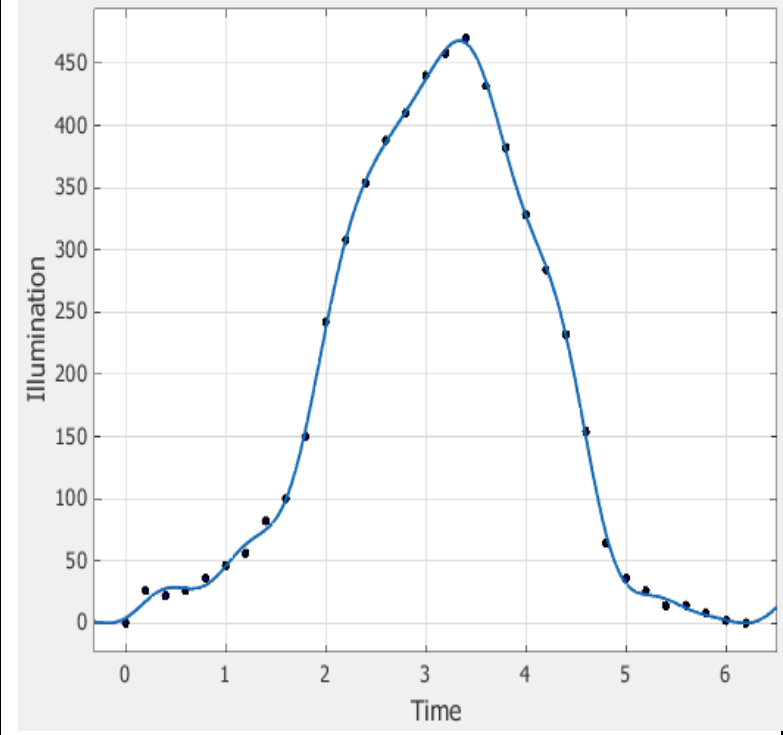

(c) $8^{\text {th }}$ Fourier Harmonic

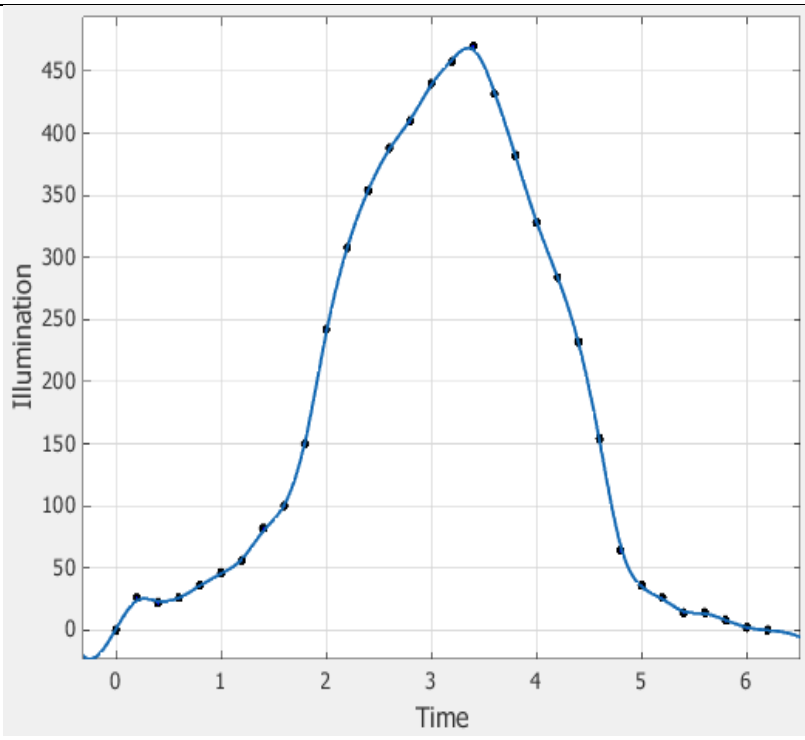

(d) Smoothing Spline 


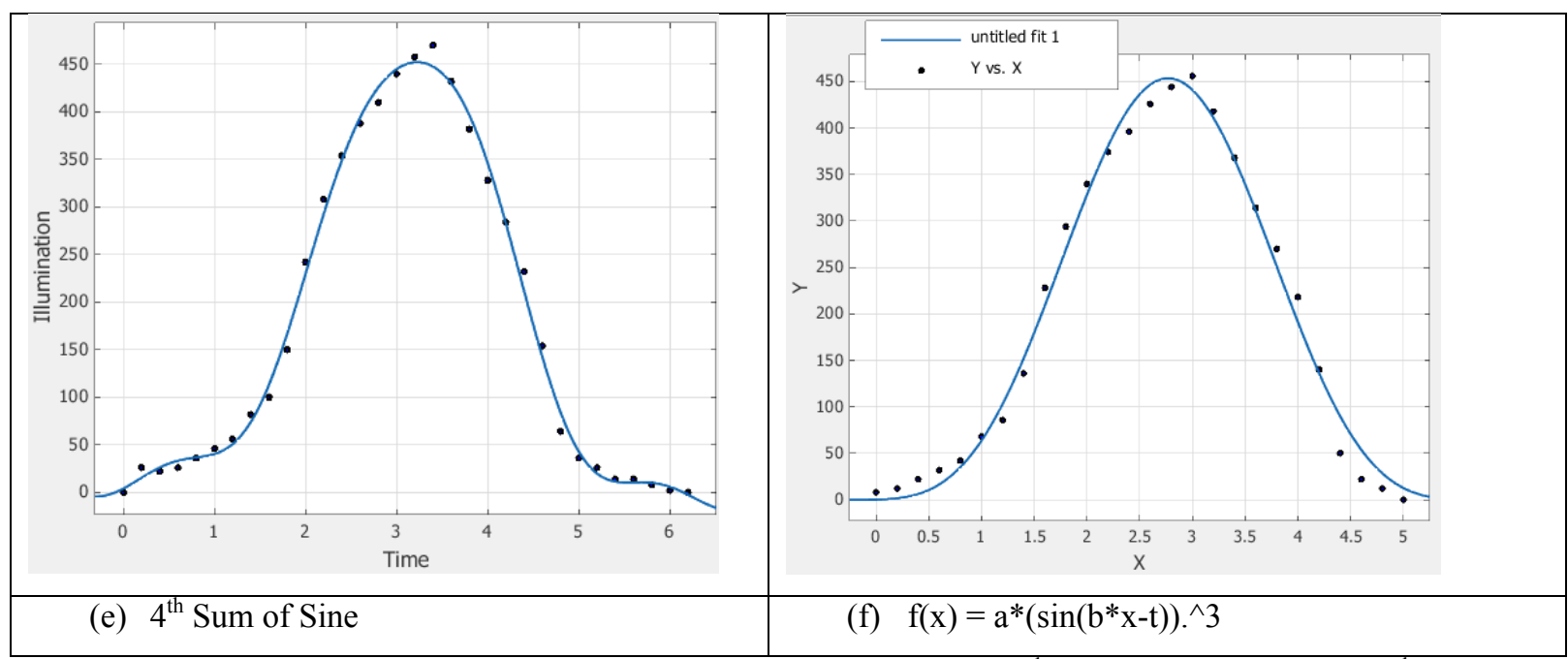

Figure 2: Prediction graphs of models tabulated in Table 2 (a) $9^{\text {th }}$ Degree Polynomial (b) $4^{\text {th }}$ Gaussian Derivative (c) $8^{\text {th }}$ Fourier Harmonic (d) Smoothing Spline (e) $4^{\text {th }}$ Sum of Sine and (f) $f(x)=a^{*}\left(\sin \left(b^{*} x-t\right)\right) \cdot{ }^{\wedge}$

\subsection{Modelling illumination due to two light sources}

In the previous section, the focus of the investigation was about finding the best mathematical model to predict illumination values variation due to a single light source. This section takes the experiments a step further towards real life scenarios by investigating the behaviour of the ALS of the handheld device in different situations under two light sources. The handheld devices can be used indoors or outdoors; under artificial lights or under natural light source and under one, two or more light sources and hence a generalisation of the successful mathematical models for more complexly illuminated environments will ultimately be required.

In these experiments the light sources were placed $50 \mathrm{~cm}$ apart at a height of approximately $75 \mathrm{~cm}$ from the mobile device, in a vertical plane of the patch of the moving mobile device.

Table 4 illustrates the accuracy of fitness obtainable by using each of the mathematical models considered in section 3.1. The best performance once again is demonstrated by the Smoothing Spline with the $8^{\text {th }}$ Fourier Harmonic and the Gaussian Derivatives demonstrating a very accurate prediction capability of over 0.99 when measured as R-square value. The accuracy of the $3^{\text {rd }}$ Sum of Sine also remains very good, being over 0.9 .

Table 4. The accuracy of prediction of Mathematical Models in illumination prediction due to two light source

\begin{tabular}{|l|l|l|}
\hline Goodness of fit/ Model & R-Square & RMSE \\
\hline $\mathrm{f}(\mathrm{x})=916^{*} \sin (0.2 * x-\mathrm{t}) .^{\wedge} 5$ & 0.7785 & 137 \\
\hline $4^{\text {th }}$ Degree Polynomial & 0.7711 & 141.9 \\
\hline $8^{\text {th }}$ Fourier Harmonic & 0.993 & 25.79 \\
\hline $8^{\text {th }}$ Gaussian Derivative & 0.9974 & 16.14 \\
\hline Smoothing Spline & 1 & 0.6761 \\
\hline $3^{\text {rd }}$ Sum of Sine & 0.9316 & 78.08 \\
\hline
\end{tabular}


Table 5 tabulates the mathematical equations for all mathematical models tested except for the Smoothing Spline that is known to be complex. When considering the complexity of implementation the $3^{\text {rd }}$ Sum of Sine appears to be the simplest, still producing a reasonable accuracy of 0.9316 .

Table 5: The mathematical models used in predicting illumination due to two light sources

\begin{tabular}{|c|c|}
\hline Mathematical Model & Equation \\
\hline Sine Function & $\mathrm{f}(\mathrm{x})=916^{*} \sin (0.2 * \mathrm{x}-\mathrm{t}) .^{\wedge} 5$ \\
\hline $4^{\text {th }}$ Degree Polynomial & $f(x)=p 1 * x^{\wedge} 4+p 2 * x^{\wedge} 3+p 3 * x^{\wedge} 2+p 4 * x+p 5$ \\
\hline $8^{\text {th }}$ Fourier Harmonic & 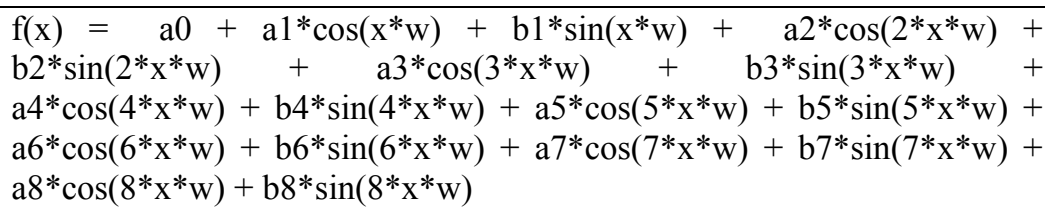 \\
\hline $8^{\text {th }}$ Gaussian Derivative & $\begin{aligned} f(x)= & a 1 * \exp \left(-((x-b 1) / c 1)^{\wedge} 2\right)+a 2^{*} \exp \left(-((x-b 2) / c 2)^{\wedge} 2\right)+ \\
& a 3^{*} \exp \left(-((x-b 3) / c 3)^{\wedge} 2\right)+a 4^{*} \exp \left(-((x-b 4) / c 4)^{\wedge} 2\right)+ \\
& a 5^{*} \exp \left(-((x-b 5) / c 5)^{\wedge} 2\right)+a 6^{*} \exp \left(-((x-b 6) / c 6)^{\wedge} 2\right)+ \\
& a 7 * \exp \left(-((x-b 7) / c 7)^{\wedge} 2\right)+a 8^{*} \exp \left(-((x-b 8) / c 8)^{\wedge} 2\right)\end{aligned}$ \\
\hline $3^{\text {rd }}$ Sum of Sine & $f(x)=a 1 * \sin (b 1 * x+c 1)+a 2 * \sin (b 2 * x+c 2)+a 3 * \sin (b 3 * x+c 3)$ \\
\hline
\end{tabular}



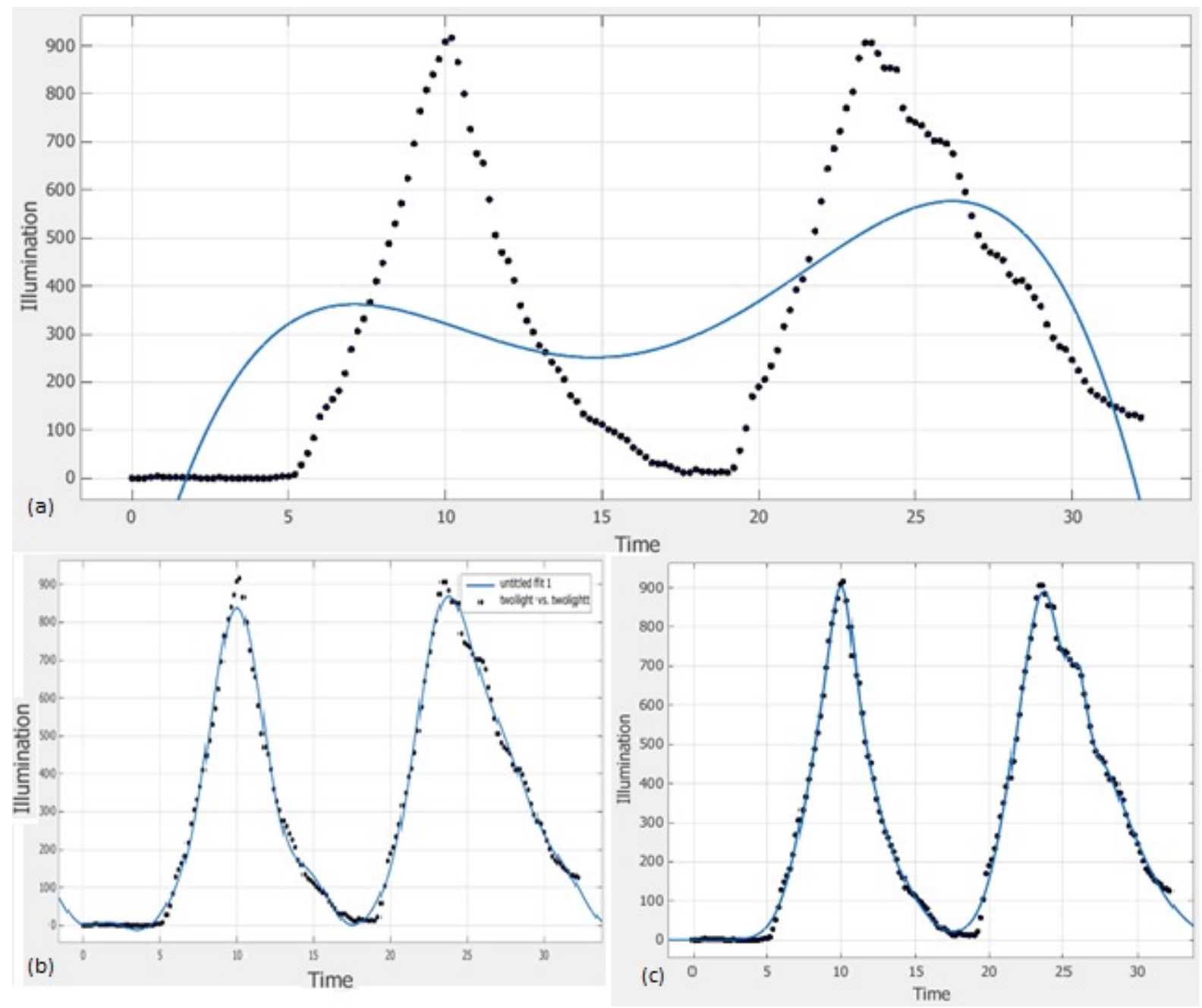

Figure 3. (a) $4^{\text {th }}$ degree of polynomial for modelling illumination due to two light sources (b) $8^{\text {th }}$ Fourier Harmonic for modelling illumination due to two light sources (c) $8^{\text {th }}$ Gaussian Derivative for modelling illumination due to two light sources

\subsection{Modelling illumination due multiple point light sources}

Table 6 compares the best accuracy values obtainable using each of the six mathematical models considered when predicting illumination distributions due to one and two point light sources. Smoothing Spline's ability to predict is maintained as $100 \%$ and the drop of accuracy of the Gaussian and Fourier models are marginal. In contrast the Sine and Polynomial models indicates a sharp decrease in accuracy. The drop of accuracy of the Sum of Sine function still remains at an acceptable level, and a viable practical solution when considering the relatively lover computational cost of its implementation when compared with the Gaussian, Fourier and Smoothing Spline alternatives.

Table 6: Comparison of performance of mathematical models for modelling illumination due to one and two point light sources

\begin{tabular}{|l|l|l|}
\hline Model /R-Square & One light source & Two light source \\
\hline Sine & 0.9893 & 0.7785 \\
\hline
\end{tabular}




\begin{tabular}{|l|l|l|}
\hline Polynomial & 0.9945 & 0.7711 \\
\hline Fourier & 0.9994 & 0.993 \\
\hline Gaussian & 0.999 & 0.9974 \\
\hline Smoothing Spline & 1 & 1 \\
\hline Sum of Sine & 0.9959 & 0.9316 \\
\hline
\end{tabular}

A final experiment was conducted using a hand held mobile device to capture ALS readings whilst the holder of the device was walking at an approximately constant speed in the corridor of a building with the phone display focussed held in a reading position. A closer inspection of the peaks of the graph and the actual physical distribution of light sources that the model developed for the two light sources above can be easily extended to cover this scenario. This is due to the observation that at any given instance when considering the practical physical separation of two light sources the device may only be affected from the illumination cause by only 2 or 3 light sources. The Fourier and Gaussian functions should provide excellent models for prediction of illumination in such environments if their practical implementation can be supported by hardware acceleration or GPUs. The Sum of Sine should still remain a reasonable model with simplicity of implementation.

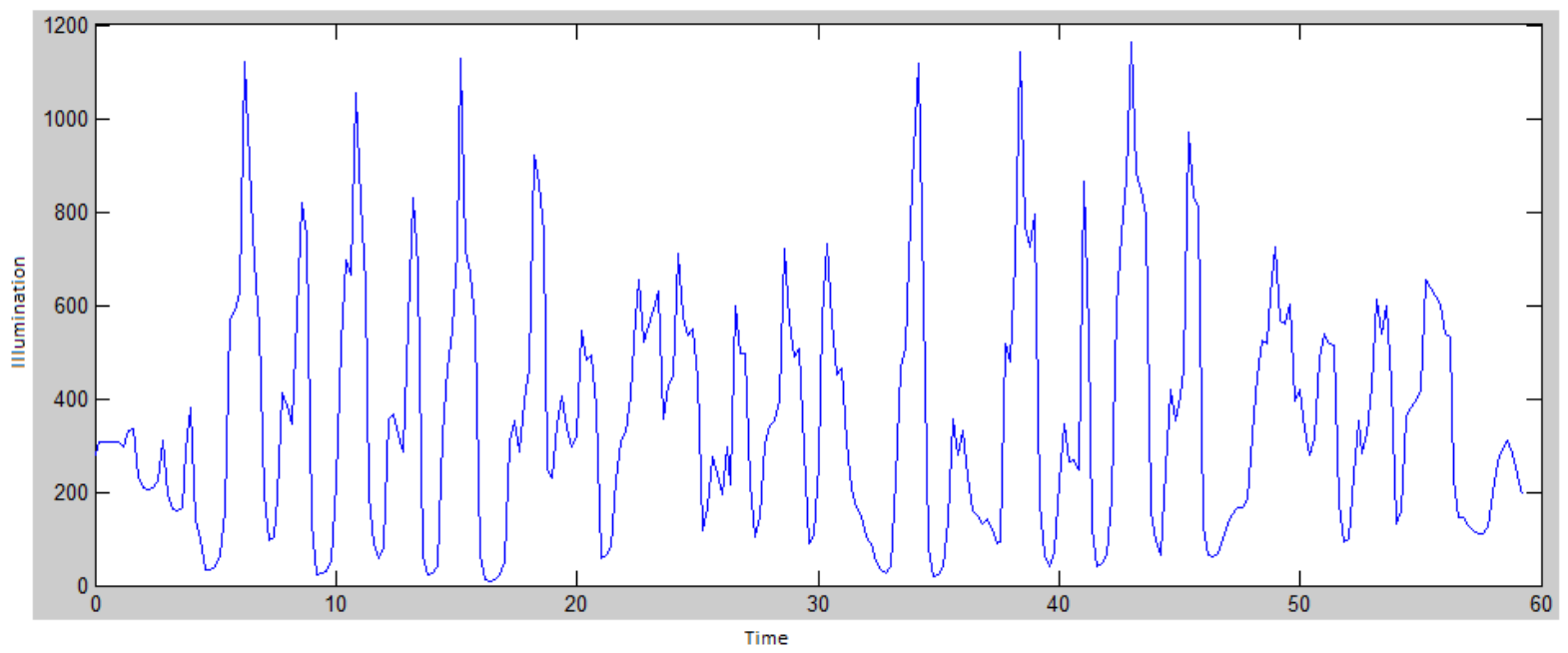

Figure 4. Illumination due to multiple point light sources

\section{CONCLUSIONS \& FUTURE WORK}

This paper has investigated the use of the capability of six mathematical models to predict the illumination variation of an environment of a mobile device that has been lit by one, two and multiple point light sources. The capability to model the illumination variations in such an environment can provide a viable solution to two of the shortcomings of modern ALS devices, namely latency and narrow angle of operation. It was demonstrated that the Smoothing Spline provides an excellent level of accuracy of prediction but with high and non-practical demand of computational requirements. Fourier harmonics and Gaussian derivatives maintained a high level of accuracy and will be viable options if efficient implementations can be realised in hardware of hardware accelerated software implementations. The simple Sum of Sine function was revealed to be reasonably accurate with low computational cost.

Our present research has been limited to modelling illumination due to uniformly distributed point light sources. In a practical environment the distribution will not be uniform and such spaces may also include diffused light sources and ambient light. Modelling of illumination distribution in such environments will need further 
investigation. However if the modelling can be performed at reasonable accuracy and computational cost it will be possible to overcome some of the shortcomings of modern ALS devices. This can have a significantly positive impact on being able to implement mobile technologies and Apps that responds to the environment and the devices usage, more efficiently and effectively.

\section{REFERENCES}

[1] Cho S, Kim Y, Lee J, Baek K, Kim S, “An Ultra-Power Ambient Light Sensor for Portable Devices.” Proc. Of SPIE Vol. 7219 72190U-1 (2009).

[2] Wikipedia, "Lux," < http://en.wikipedia.org/wiki/Lux>, (26 February 2015).

[3] Wikipedia, "Backlight," $<$ http://en.wikipedia.org/wiki/Backlight >, (5 February 2015).

[4] Bai, C.-H. C. Y.-W. "Using fuzzy logic and light-sensor for automatic adjustment of backlight brightness in a mobile computer." IEEE DOI: 10.1109/ISCE.2012.6241719 (2012).

[5] Ma, T.-Y., Lin, C.-Y., Hsu, S.-W., Hu, C.-W., \& Hou, T.-W., "Automatic brightness control of the handheld device display with low illumination." IEEE International Conference on Computer Science and Automation Engineering (CSAE), 382-385. doi:10.1109/CSAE.2012.6272797 (2012).

[6] Mailloux, J., "Automatic screen and keypad brightness adjustment on a mobile handheld electronic device." Patente: US 7701434 B2, (2013).

[7] Dalen, V., “Indoor/outdoor detection.” Patente: US 8592744 B2 (2010).

[8] Robinson, A., Ca, E., \& Crugnale, T. J. "Multiple orientation mobile electronic handheld device and method of ambient light sensing and backlight adjustment implemented therein." Paatente: US 8508465 B2 (2013).

[9] Wikipedia, "Inverse Square Law." < http://en.wikipedia.org/wiki/Inverse-square_law >, (15 March 2015).

[10] Carl de Boor, [A Practical Guide to Splines], Applied Mathematical Sciences 27, Springer-Verlag, 235-276 (1978).

[11] Wikipedia, "Coefficient of determination", < http://en.wikipedia.org/wiki/Coefficient_of_determination >, (21 February 2015)

[12] Wikipedia, "Spline (Mathematics)", < http://en.wikipedia.org/wiki/Spline_\%28mathematics\%29>, (15 March 2015) 